

\section{Evaluation of the health and physical activity characteristics of undergraduate paramedic and nursing students}

Peter S Micalos PhD, is Lecturer ${ }^{1}$; Alexander J MacQuarrie MBA, is Lecturer ${ }^{1}$; Brian A Haskins MHIth Mgt, is Lecturer ${ }^{1}$; Eileen Barry BSc, is Lecturer'; Judith K Anderson PhD, is Senior Lecturer ${ }^{2}$

\section{Affiliations:}

${ }^{1}$ School of Biomedical Sciences, Charles Sturt University, Australia

${ }^{2}$ School of Nursing, Midwifery, and Indigenous Health, Charles Sturt University, Australia

\section{Abstract}

\section{Introduction}

Levels of physical activity and health status are substantially influenced during the early adulthood years. The purpose of this research was to evaluate the health and physical activity characteristics of undergraduate paramedic and nursing students.

\section{Methods}

This research comprised a descriptive and inter-discipline survey of a sample of university students enrolled in healthcare programs. The health status and physical activity patterns of paramedic and nursing undergraduate students were assessed using the 36-item Short Form Health Survey (SF-36) and the International Physical Activity Questionnaire (IPAQ), respectively. Statistical comparisons for health status - total, eight health dimensions and weekly metabolic equivalent were performed.

\section{Results}

A total of 226 undergraduate students were surveyed with $66.4 \%$ enrolled in the paramedic, $17.7 \%$ in the nursing, and $15.9 \%$ enrolled in the double degree nursing/paramedic Bachelor programs. Results for the SF-36 total show a significant difference in health status between the undergraduate programs $(p=0.001)$. Reduced health dimensions were also revealed among first year students. Results for the IPAQ shows a significant difference in physical activity between the programs $(p=0.03)$. Comparison between IPAQ and year of study show a significant difference between groups $(p=0.03)$.

\section{Conclusion}

Substantially reduced health status and physical activity patterns were shown in nursing students compared to paramedic students and among first year university undergraduates. This research highlights the need to further investigate the health status and physical activity patterns within university healthcare students.

Keywords:

health status, mental health, vitality

Corresponding author: Peter S Micalos, pmicalos@csu.edu.au 


\section{Introduction}

Students enrolled in Australian higher education constitute a large transient population that go on to exert influence in future professional careers and in key roles within society. Levels of physical activity and health status are substantially influenced during these early adulthood years (1). Australian university students show increased potential to meet exercise guidelines and are less likely to be current smokers compared to nonhigher education students (2). However, a significant decrease in mental health and excessive levels of fatigue have been shown in university students (3). Declining mental health and physical activity patterns have been revealed among university students in the United States (US) (4). Reduced levels of physical activity in older ( $\geq 20$ years) compared to younger ( $\leq 19$ years) university students have previously been reported (5). Engagement in regular physical activity is important in reducing the risk of chronic lifestyle diseases and disability later in life $(6,7)$.

It is important to monitor trends in health and physical activity patterns in early adult life as this represents a period in which health behaviours become established and physical inactivity can manifest $(7,8)$. Previous research shows increased levels of fatigue are linked to reduced academic outcomes in over half the surveyed university students $(3,9)$. The implications for this are that students who became physically inactive show reduced levels of vigour, which adversely impacts on retention and performance $(10,11)$. Changes in levels of physical activity during university studies may reflect disruptions in established living patterns that persist into the workforce (12). Moreover, the promotion of university student health has been shown to enhance future wellbeing and career performance (13).

Presently there is limited information on the health status of the undergraduate student population in Australia. Differences in health and physical activity patterns have been revealed between academic disciplines and gender among Spanish university students (14). Increased sedentary rates have been shown in first year university Basque Country nursing students compared to other matched university students (15). Additionally, reduced emotional wellbeing (16) and decreased health-related quality of life (17) have been revealed among university medical students. Together, these studies suggest that the health status and level of physical activity may substantially differ among university students enrolled in healthcare professions. Currently there is a deficit of information on the health status and physical activity of paramedic students during their studies at university. In the university context, additional challenges faced by many students include adaptations to independent living. The purpose of this study is to evaluate the health and physical activity patterns of Australian university students enrolled in undergraduate paramedic and nursing programs.

\section{Methods}

\section{Study design}

This research comprised a descriptive and inter-discipline survey of a sample of university students enrolled in healthcare programs.

\section{Participants}

Students enrolled in undergraduate paramedic, nursing and double degree nursing/paramedic programs within a regional Australian university were invited to participate in the study. Data on participant characteristics included age, height, weight, gender, home postcode, currently living (on campus, off-campus, at home), program studied (paramedic, nursing, paramedic/nursing double degree), year of study at university (ie. first, second, third, fourth), health status and physical activity.

\section{Instrumentation}

Assessment of health status and physical activity were performed via the 36-item Short Form Health Survey (SF-36) (18) and International Physical Activity Questionnaire (IPAQ) $(19,20)$. The SF-36 health questionnaire shows a percentage total health score (SF-36-total). Additional health dimensions assessed in the SF-36 include physical functioning, social functioning, role limitations physical, role limitations emotional, mental health, vitality, pain and general health.

The IPAQ involves the summation of self-reported levels of physical activity associated with vigorous-intensity, moderateintensity and walking per week. The IPAQ scoring reveals weekly energy expenditure for physical activity (IPAQ-total) as multiples of the resting metabolic equivalent (MET)-minutes per week. Threshold levels of physical activity are categorised as 'low' (less than $600 \mathrm{MET}$-min/week), 'moderate', and 'high' (at least $1500 \mathrm{MET}$-min/week) (19).

\section{Data analysis}

Statistical procedures included descriptive, ANOVA, correlation and chi-square analyses. Comparisons were performed for health status and physical activity between university healthcare programs and year of study. Initial checks for differences between gender with health and physical activity were also performed. Post hoc least significant difference procedure was performed for multiple comparisons (less than six groups). Checks for equality of error variances were performed and non-parametric (21) tests were applied where required. Effect sizes were determined by Cohen's $d$ to assess magnitude of differences in health status of students living at home compared to campus and off-campus facilities. Results are presented as mean \pm standard error, unless indicated. The threshold for significance was set at $p<0.05$. 


\section{Ethics}

Ethics approval from Charles Sturt University Minimal Human Ethics Committee was obtained (Protocol Number 406/2013/05).

\section{Results}

\section{Descriptive}

From the total pool of 722 enrolled on-campus undergraduate paramedic and nursing students, $226(31 \%)$ completed the survey. Results from the surveyed participants showed that $66.4 \%$ were enrolled in the paramedic course, $17.7 \%$ were enrolled in the nursing course, and $15.9 \%$ of were enrolled in the double degree paramedic/nursing program. The mean age and gender profile for each program surveyed is shown in Table 1. The percentage surveyed respondents included $37.4 \%$ in first year, $37 \%$ in second year, $19.9 \%$ in third year and $5.7 \%$ in fourth year. From the total surveyed students, $45.7 \%$ lived on-campus while $43.8 \%$ and $10.6 \%$ indicated they lived off-campus, or at home, respectively.

Table 1. The mean age and gender profile of the university undergraduate healthcare students surveyed

\begin{tabular}{|c|c|c|c|}
\hline & $\begin{array}{l}\text { Number of } \\
\text { respondents }\end{array}$ & $\begin{array}{l}\text { Mean age } \\
\text { (yrs } \pm S D \text { ) }\end{array}$ & Gender \% \\
\hline \multirow{2}{*}{ Paramedic } & \multirow{2}{*}{150} & \multirow{2}{*}{$23.5 \pm 8.9$} & $59 \%$ male \\
\hline & & & $41 \%$ female \\
\hline \multirow{2}{*}{ Nursing } & \multirow{2}{*}{40} & \multirow{2}{*}{$31.1 \pm 11$} & $25 \%$ male \\
\hline & & & $75 \%$ female \\
\hline \multirow{2}{*}{$\begin{array}{l}\text { Paramedic/ } \\
\text { nursing }\end{array}$} & \multirow{2}{*}{36} & \multirow{2}{*}{$23.9 \pm 8.9$} & $39 \%$ male \\
\hline & & & $61 \%$ female \\
\hline
\end{tabular}

\section{Health status}

Comparison between SF-36-total and gender did not show significant difference between males and females $(p=0.097)$. Results for health status show that there was a significant difference $(p=0.001)$ in SF-36-total health score between the three groups comprising the paramedic, nursing and double degree paramedic/nursing programs (Figure 1A). Post hoc analysis shows a significant difference between paramedic and nursing students $(p=0.001)$ and between nursing students and double degree paramedic/nursing students $(p=0.001)$.

Comparison between SF-36-total health score and year of study (Figure 1B), shows no difference between groups $(p=0.136)$. Comparisons (21) between the SF-36 health dimensions and year of study (Figure 2 ) reveal a significant difference in vitality $(p=0.013)$, social functioning $(p=0.049)$, and mental health-total scores $(p=0.039)$. Post hoc analysis shows significant difference in vitality between year 1 and 3 $(p=0.010)$, year 1 and $4(p=0.027)$, year 2 and $3(p=0.024)$, a significant difference in social functioning between year 1 and $2(p=0.019)$, year 1 and $3(p=0.043)$, and in mental health-total between year 1 and $3(p=0.015)$.

There was a small to medium effect size difference (Cohen's d) between SF-36-total health status for students living oncampus and off-campus facilities compared to living at home $(0.23$ and 0.48 , respectively), although this difference was not statistically significant $(p=0.07)$.

\section{Physical activity patterns}

Comparison between IPAQ-total and gender did not show significant difference between males and females $(p=0.94)$. Results of comparisons between IPAQ-total and the paramedic, nursing and double degree nursing/paramedic programs (Figure $3 \mathrm{~A}$ ) show a significant difference in physical activity patterns $(p=0.030)$. Post hoc analysis shows a significantly reduced level of physical activity among nursing students compared to paramedic students $(p=0.009)$, and between nursing and nursing/paramedic double degree students $(p=0.044)$.

Significant differences (21) were revealed between IPAQ-total and year of study $(p=0.030)$. Post hoc revealed significant difference between year 1 and 3 for IPAQ-total $(p=0.007)$. No statistical difference was found between year 3 and $4(p=0.14)$ and year $1-4(p=0.448)$. Comparison between currently living and IPAQ-total found no significant difference between groups $(p=0.880)$.

Pearson chi-square analysis between physical activity (low, moderate, high) and year of study (first, second, third, fourth) shows a significant difference between groups $(p=0.01)$ with $26.6 \%$ of first year, $25 \%$ of second year and $7 \%$ of third and fourth year reporting low physical activity levels (<600 MET$\mathrm{min} /$ week).

\section{Discussion}

The present study reveals a significant difference in the health status between paramedic, nursing and double-degree paramedic/nursing programs. These results indicate a reduced health status among students enrolled in nursing compared to students enrolled in the paramedic program. Additionally, a reduced level of physical activity was revealed among nursing students compared to paramedic students. The implication of this is that approaches to health and physical activity interventions could be applied to encourage health awareness at a program level. This is noteworthy as deficiencies in health and physical activity among university healthcare students may contribute to reduced competency in future workplace demands and diminished health outcomes later in life $(22,23)$. 
A)

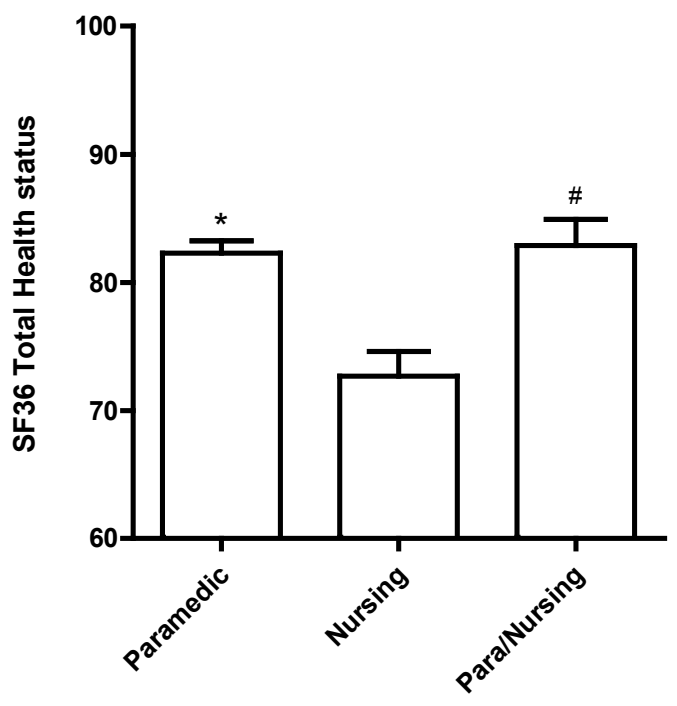

B)

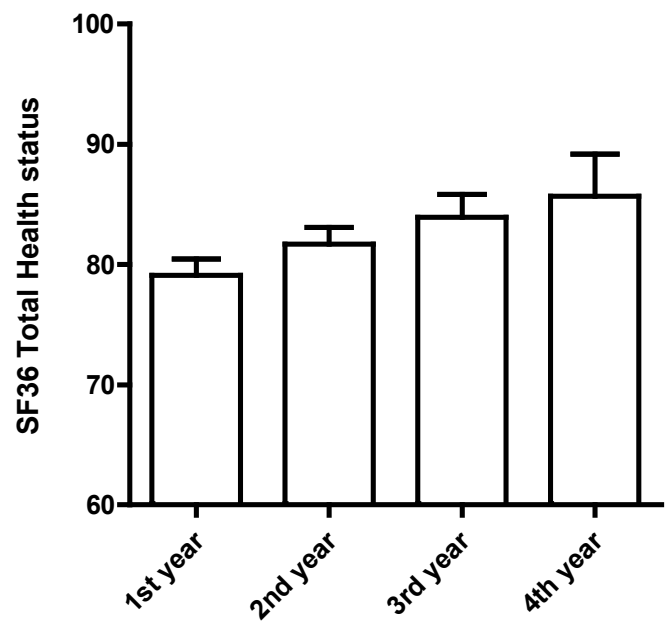

Figure 1. Results for undergraduate student SF-36-total health scores are shown for the university healthcare programs surveyed $(A)$, and between undergraduate year of study $(B)$. Significant differences $(p<0.05)$ are shown between paramedic and nursing students ${ }^{*}$, and between double degree paramedic/nursing and nursing students\# 


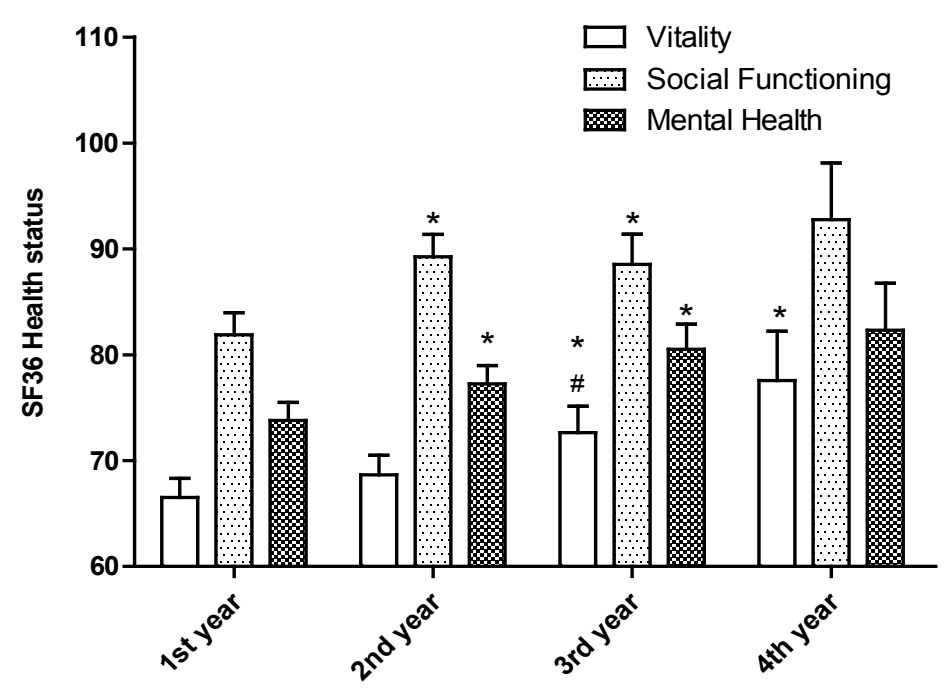

Figure 2. Results for the SF-36 health dimensions of vitality, social functioning and mental health-total for each undergraduate year of study

*Significant differences compared to first year $(p<0.05)$

\#Significant difference $(p<0.05)$ between second and third year

A)

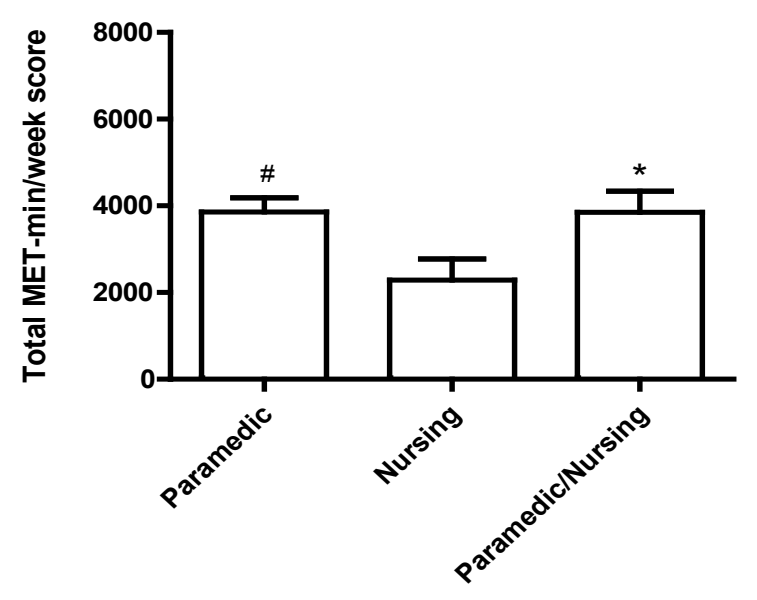

B)

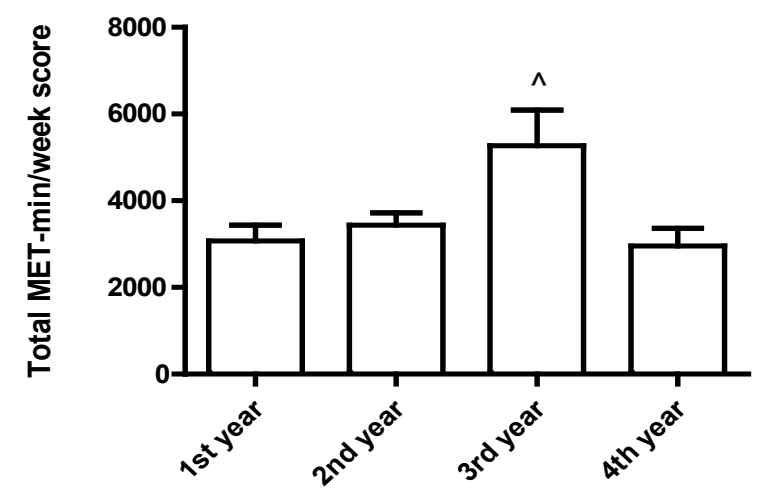

Figure 3. Results showing student physical activity patterns (IPAQ-total) between healthcare programs (A) and year of study $(B)$. Significant differences $(p<0.05)$ are shown between paramedic and nursing students\#, between double degree paramedic/nursing and nursing students, ${ }^{*}$ and among the years of study 
University students often encounter significant challenges associated with the transition into academic routine (11). These challenges significantly impact on the health status of university students. A sense of 'feeling overwhelmed' among US university students has been trending upward in first year students with fewer students reporting above average emotional health (24). Previous research confers that first year students report the highest levels of psychological distress of any undergraduate year and are at risk of compromised psychological wellbeing (25). In accord with this, the present results show that first year university students report reduced health dimensions in vitality, social functioning, and mental health compared to the later years. Additionally, previous research highlights concerns raised by students on the stigma and anonymity in seeking health counselling among rural Australian university students (3). On this basis, it is possible that the reduced health dimensions in rural first year students may reflect a transition period into university studies that may be associated with differences in seeking professional health advice.

The present results show a low to moderate effect size of increased health status for students living on-campus and in off-campus university living facilities compared to students living at home. However, there was no difference in levels of physical activity between these groups. A possible reason for the increased health status in students living in university facilities compared to students living at home is the accessibility to campus support services. Additionally, the potential for enhanced levels of social interaction within the campus lifestyle and development of independent living skills may contribute to enhanced health outcomes for students living on-campus and in off-campus living facilities. Further research is required to reveal the differences in health status among students living at home compared to students living in university campus-related facilities.

The present results also show reduced levels of physical activity in first year university students. These results confer with previous studies showing reduced physical activity in first year university students (9). However, our data also show a significant increase in levels of physical activity in third year university students. Possible reasons for the increase in physical activity in third year are that university study schedules and available leisure time may substantially differ between the years of study. Our results also show reduced levels of physical activity in nursing compared to paramedic students. This is aligned with previous research showing reduced physical activity in nursing students compared to other university cohorts (15).
The reason for the reduced physical activity level among university students may be associated with student attitudes to health and physical activity. Previous research on university medical students shows that student knowledge and attitudes to health and exercise represent strong predictors of physical activity (27). Presently, some paramedic organisations require physical fitness testing as part of the recruitment and selection process, however, there is no standard physical fitness requirement across various university programs. It is uncertain as to the extent this impacts on the physical activity levels of these students. Following a campus-based intervention program, significantly increased levels of physical activity and long-term health benefits were shown among university students (27). Further research on student attitudes is required to elucidate differences in health and physical activity patterns between university cohorts.

\section{Limitations}

This study adds to the limited information available on the health and physical activity profile of undergraduate students enrolled in healthcare programs. These findings merit further research into student mental health between university cohorts. Limitations in the present study are that demographic data on academic performance, socio-economic, nutrition, substance abuse and sleep patterns were not assessed. We also had reduced sample size in the fourth year undergraduate data. Additionally, comparisons of students with English as a second language, at metropolitan universities and other programs could further glean information about the health and physical activity patterns among university students. It should also be noted that the current data is based on self-report method, which has the potential for bias.

\section{Conclusion}

The present results show significant differences in undergraduate nursing student health and physical activity compared to paramedic students and double-degree nursing/ paramedic students. Reduced health components were also shown in first year undergraduate students compared to years 2, 3 and 4. Strategic interventions could target health within university programs and offer an important avenue for maintaining health attitudes and behaviours when entering a professional career.

\section{Acknowledgements}

We wish to thank Mr Mark Curran in enabling the availability of facilities for research data collection. 


\section{References}

1. Sawyer SM, Afifi RA, Bearinger LH, et al. Adolescence: a foundation for future health. Lancet 2012;379:1630-40. doi: http://dx.doi.org/10.1016/S0140-6736(12)60072-5

2. ABS. (2013). Hitting the books: Characteristics of higher education students. Australian Bureau of Statistics. Available at: www.abs.gov.au/AUSSTATS/abs@.nsf/Lookup/4102.0Ma in+Features20July+2013\#p7

3. Hussain R, Guppy M, Robertson S, Temple E. Physical and mental health perspectives of first year undergraduate rural university students. BMC Public Health 2013;13:848. doi: 10.1186/1471-2458-13-848

4. Eisenberg D, Gollust SE, Golberstein E, Hefner JL. Prevalence and correlates of depression, anxiety, and suicidality among university students. Am J Orthopsychiatry 2007;77:534-42. doi: 10.1037/0002-9432.77.4.534

5. Huang TTK, Harris KJ, Lee RE, Nazir N, Born W, Kaur H. Assessing overweight, obesity, diet, and physical activity in college students. J Am Coll Health 2003;52:83-86. doi: 10.1080/07448480309595728

6. Australian Institute of Health and Welfare. Risk factors contributing to chronic disease. Canberra: AlHW, 2012.

7. Keating XD, Guan J, Pinero JC, Bridges DM. A metaanalysis of college students' physical activity behaviors. J Am Coll Health 2005;54:116-25. doi: 10.3200/JACH.54.2.116126

8. Haase A, Steptoe A, Sallis JF, Wardle J. Leisure-time physical activity in university students from 23 countries: associations with health beliefs, risk awareness, and national economic development. Prev Med 2004;39:182-190. doi: http://dx.doi.org/10.1016/j.ypmed.2004.01.028

9. Bray SR, Born HA. Transition to university and vigorous physical activity: Implications for health and psychological well-being. J Am Coll Health 2004;52:181-8. doi: 10.3200/ jach.52.4.181-188

10. Bray SR, Kwan MY. Physical activity is associated with better health and psychological well-being during transition to university life. ibid. 2006;55:77-82. doi: 10.3200/ jach.55.2.77-82

11. Melnyk B, Kelly S, Jacobson D, Arcoleo K, Shaibi G. Improving physical activity, mental health outcomes, and academic retention in college students with Freshman 5 to thrive: COPE/Healthy lifestyles. J Am Assoc Nurse Pract 2014;26:314-22. doi: 10.1002/2327-6924.12037

12. Dishman RK, Dunn AL. Exercise adherence in children and youth: implications for adulthood. In: Dishman RK, editor. Exercise adherence: its impact on public health (pp. 155-200). Champaign, IL: Human Kinetics, 1988.

13. Bailey R, Hillman C, Arent S, Petitpas A. Physical activity: An underestimated investment in human capital? J Phys Act Health 2013;10:289-308.
14. Varela-Mato V, Cancela JM, Ayan C, Martin V, Molina A. Lifestyle and health among Spanish university students: differences by gender and academic discipline. Int $\mathrm{J}$ Environ Res Public Health 2012;9:2728-41. doi: 10.3390/ ijerph9082728

15. Irazusta A, Gil S, Ruiz F, et al. Exercise, physical fitness, and dietary habits of first-year female nursing students. Biol Res Nurs 2006;7:175-86.

16. Parkerson GRJ, Broadhead WE, Tse CK. The health status and life satisfaction of first-year medical students. Acad Med 1990;65:586-8.

17. Paro HB, Morales NM, Silva CH, et al. Health-related quality of life of medical students. Med Educ 2010;44:227-35. doi: 10.1111/j.1365-2923.2009.03587

18. Jenkinson C, Coulter A, Wright L. Short form 36 (SF36) health survey questionnaire: normative data for adults of working age. BMJ (Clinical Research Edition) 1993;306:1437-40.

19. IPAQ. (2005). Guidelines for data processing and analysis of the International Physical Activity Questionnaire (IPAQ). Available at: www.ipaq.ki.se/scoring.pdf

20. Craig CL, Marshall AL, Sjöström M, et al. International Physical Activity Questionnaire: 12-Country reliability and validity. Med Sci Sports Exer 2003;35;1381-95.

21. McCrum-Gardner, E. Which is the correct statistical test to use? Br J Oral Maxillofac Surg 2008;4:38 -41. doi: http:// dx.doi.org/10.1016/j.bjoms.2007.09.002

22. Chapman D, Peiffer J, Abbiss C, Laursen P. A descriptive physical profile of Western Australian male paramedics. J Emerg Prim Health Care 2007;5:Article 2.

23. Hansen D, Vranckx P, Broekmans T, et al. Physical fitness affects the quality of single operator cardiocerebral resuscitation in healthcare professionals. Eur J Emerg Med 2012;19:28-34. doi: 10.1097/MEJ.0b013e328347a2aa

24. Pryor JH, Hurtado S, DeAngelo L, Palucki B, Tran S. (2010). Incoming college students rate emotional health at record low, annual survey finds (UCLA Graduate School of Education and Information Studies, Trans.) The American Freshman: National Norms for Fall 2010 The Higher Education Research Institute.

25. Adlaf EM, Gliksman L, Demers A, Newton-Taylor B. The prevalence of elevated psychological distress among Canadian undergraduates: findings from the 1998 Canadian Campus Survey. J Am Coll Health 2001;50:67-72. doi: 10.1080/07448480109596009

26. Liang MT, Dombrowski HT, Allen TW, et al. Do medical students' knowledge and attitudes about health and exercise affect their physical fitness? J Am Osteopath Assoc 1993:93:1020-4.

27. Leslie E, Fotheringham M, Owen N, Veitch J. A university campus physical activity promotion program. Health Promot J Aust 2000;10:51-4. 